\title{
Cellular Automaton Study of Time-Dynamics of Avalanche Breakdown in IMPATT Diodes
}

\author{
G. ZANDLER ${ }^{\mathrm{a}}$, R. OBERHUBER ${ }^{\mathrm{a}}$, D. LIEBIG $^{\mathrm{a}}, \mathrm{P}^{\mathrm{P}}$ VOGL $^{\mathrm{a}, *}$, \\ M. SARANITI ${ }^{b}$ and P. LUGLI ${ }^{c}$ \\ ${ }^{a}$ Walter Schottky Institute, Technical University of Munich, 85748 Garching, Germany; \\ ${ }^{\mathrm{b}}$ Electrical Engineering Department, Arizona State University, Tempe, AZ 85287-6206; \\ ${ }^{c}$ Electrical Engineering Department, University of Rome, Tor Vergata, 00133 Rome, Italy
}

\begin{abstract}
Employing a recently developed efficient cellular automaton technique for solving Boltzmann's transport equation for realistic devices, we present a detailed study of the carrier dynamics in GaAs avalanche $p-i-n$ (IMPATT) diodes. We find that the impact ionization in reverse bias $p-i-n$ diodes with ultrathin (less than $50 \mathrm{~nm}$ ) intrinsic regions is triggered by Zener tunneling rather than by thermal generation. The impact generation of hot carriers occurs mainly in the low-field junction regions rather than in the high field intrinsic zone. The calculations predict significantly more minority carriers on the $n$-side than on the $p$-side.
\end{abstract}

Keywords: Cellular Automata, IMPATT diodes, zener tunneling, impact ionization, avalanche breakdown

\section{INTRODUCTION}

The carrier dynamics in modern nanometer size devices is dominated by hot carrier effects and nonlocal transport phenomena such as tunneling and impact ionization. A realistic prediction and understanding of these effects in real devices that operate at room temperature requires at least the solution of the full semiclassical Boltzmann equation. A few years ago, we have developed a novel method, the Cellular Automaton (CA) approach namely, that provides a computationally efficient scheme for solving Boltzmann's equation in position and momentum phase space [1-2]. Since then, we have significantly refined this approach [3-4] and enhanced its speed and robustness; a detailed review of the present status of the CA method has been given very recently [5]. The present paper focuses on the application of this scheme to a physically intriguing situation where the carrier dynamics is highly complex and very far from equilibrium. We present a micro-

\footnotetext{
*Corresponding author: phone: +49-89-289-12750, FAX +49-89-289-12737, email: vogl@wsi.tu-muenchen.de.
} 
scopic analysis of the carrier dynamics near avalanche breakdown in GaAs and $\mathrm{AlGaAs}$ IMPATT diodes.

\section{THE CA METHOD}

We briefly summarize the highlights of the CA method [1-5]. A cellular automaton consists of a lattice with a finite number of states attached to each lattice site that can be interpreted as pseudoparticles. Their dynamics is governed by a limited set of local transition rules. The perhaps most crucial factor that sets cellular automata apart from standard finite differencing methods is the reduction of all physical variables to a finite set of discrete values. In contrast to finite differencing methods, the size of this set can be kept relatively small due to an optimized phase space discretization. This discretization utilizes the fact that the final carrier distribution function changes on a much coarser scale in phase space than typical changes of phase space variables within on time step. In our present implementation, we use a two-dimensional hexagonal lattice in real space. Attached to each lattice site are of the order of $10^{5}$ momentum states that form a hexagonal closed packed structure. Transition rules among these states represent the quantum mechanical collisions as well as the drift and diffusion terms in the Boltzmann equation. In the simulation, the particle dynamics consists mainly in look-up operations of pre-calculated and hierarchical scattering tables. This results in a numerically very efficient algorithm for the particle dynamics that is typically faster than the standard Monte Carlo scheme by a factor of $30-50$.

From a physics point of view, in particular concerning scattering mechanisms, the $\mathrm{CA}$ and Monte Carlo scheme are equivalent. Indeed, we have employed both methods in obtaining the results of this paper, mostly to check the accuracy and consistency of the CA results.

\section{CARRIER DYNAMICS IN IMPATT DIODES}

Recently, GaAs and GaAlAs based IMPact Avalanche Transit Time (IMPATT) diodes have been fabricated with a high power output at frequencies up to $200 \mathrm{GHz}$ [ 6-8]. The avalanche zone of such a diode consists of a $p-i-n$ diode with highly doped $n$ and $p$ regions and an ultrathin intrinsic zone of 20 to $50 \mathrm{~nm}$. The build-up of the carrier avalanche under high reverse bias is a subtle interplay between thermal generation, interband (Zener) tunneling, impact ionization, and other scattering mechanisms [9].

\subsection{Scattering Rates}

The present simulations incorporate all relevant standard scattering mechanisms for carriers, such as ionized impurity, plasmon, intra- and intervalley phonon scattering, alloy scattering, and thermal generation, and nonparabolic electron and hole bands [10]. Impact ionization is accounted for by invoking the model of Kane [11], using density of states that have been calculated with the empirical pseudopotential method. The $k \cdot p$ model of Krieger [12] is employed to calculate the interband tunneling rate for direct transitions as a function of electric field. The absolute magnitude of the impact ionization rate of electrons and holes is chosen as to reproduce the experimental ionization coefficients in bulk GaAs and $\mathrm{AlGaAs}$ [13-15].

In Figures 1 and 2, we show several scattering rates for electrons and holes in GaAs. This figure reveals that the impact ionization scattering rate for holes is markedly lower than for electrons. An interesting consequence of this difference is illustrated in Figure 2. It shows the average distance an electron or hole travels in bulk GaAs between impact ionization scattering events when the field is $1 \mathrm{MV} / \mathrm{cm}$. In contrast to the quasi-ballistically moving electrons, the slower holes suffer many other scattering processes before they are able to 


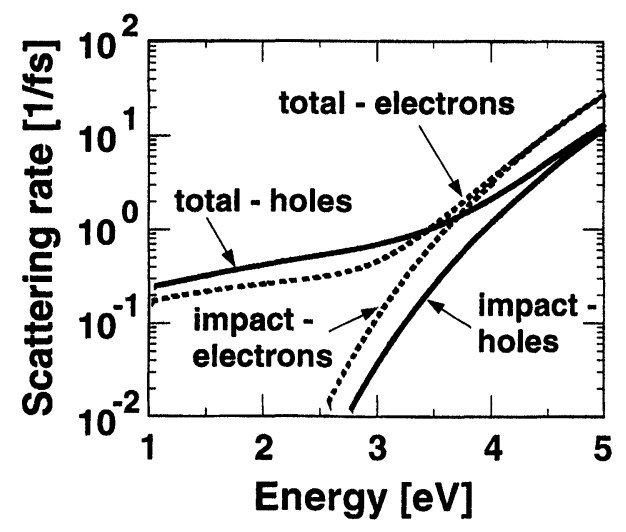

FIGURE 1 Impact ionization scattering rates (labeled by "impact") and total scattering rates ("total"), in units of $1 / \mathrm{fs}$, for electrons in the $\Gamma$ valley and heavy holes, respectively, in bulk GaAs at room temperature and a doping level of $2 \times 10^{18} \mathrm{~cm}^{-3}$ as a function of energy in $\mathrm{eV}$.

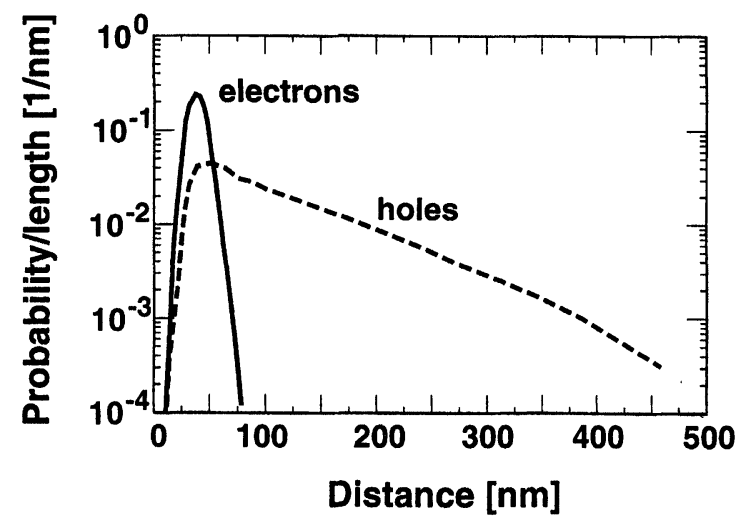

FIGURE 2 Probability per distance for an electron (respectively, hole) in bulk GaAs to initiate an impact ionization along its trajectory of a length specified by the abscissa in $\mathrm{nm}$. The electric field is $1 \mathrm{MV} / \mathrm{cm}$.

impact ionize. This leads to the broad distribution of hole trajectories in Figure 2.

\subsection{The Avalanche Generation Process: Qualitative Picture}

We now consider a GaAs $p$-i-n diode with a $20 \mathrm{~nm}$ intrinsic zone and a carrier concentration of $n=p=2 \times 10^{18} \mathrm{~cm}^{-3}$. The density in the intrinsic zone is set to $n=2 \times 10^{15} \mathrm{~cm}^{-3}$. In Figure 3, we illustrate schematically the time development of

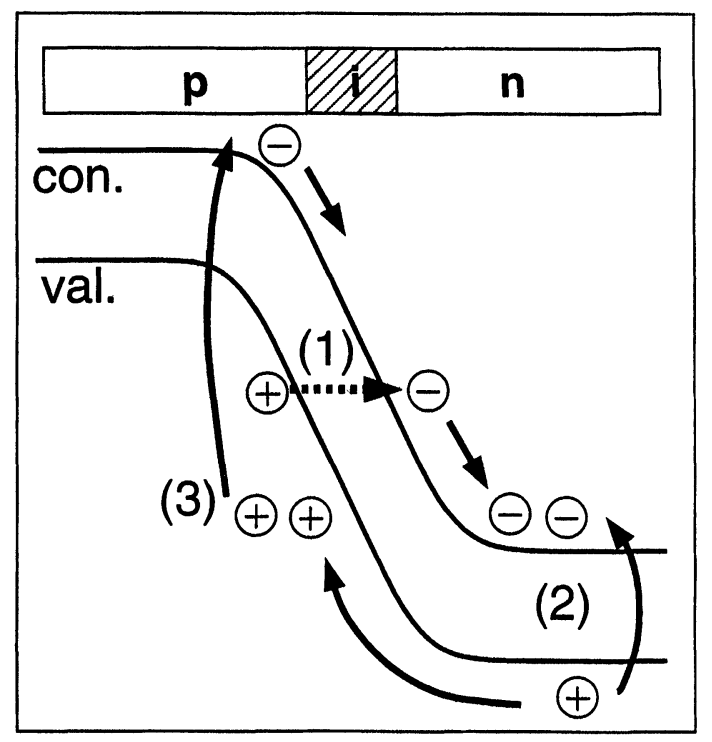

FIGURE 3 Schematic picture of carrier dynamics in a highly doped IMPATT diode with ultrathin $i$-zones under reverse bias that is close to breakdown. The relevant dynamical processes are Zener tunneling, labeled by (1), impact ionization in the depletion zone (labeled by (2) and only shown on the $n$-side for simplicity), and impact ionization in the high field region, labeled by (3).

the carrier distribution in the diode after applying a reverse bias of $5.5 \mathrm{eV}$.

For a reverse bias between 4 and $5 \mathrm{~V}$, the resulting high field of approximately $1 \mathrm{MV} / \mathrm{cm}$ initiates electron hole pair generation by interband tunneling (step (1) in Fig. 3). The thermal generation rate is found to be negligible compared to the tunneling rate for this device geometry. The generated electrons on the $n$-side get rapidly accelerated by the electric field, move away from the band edge and gain a significant amount of energy. The key point is that the electrons dissipate their excess energy efficiently by impact ionization within the low field depletion region rather than in the high field zone (step (2)). The holes that are generated via these impact ionization processes get accelerated by the field back into and through the whole intrinsic zone. This allows the holes to gain sufficient excess energy so that they induce impact ionization processes already within the $i$-zone. This ignites the avalanche process (step (3)). 
Thus, the carrier generation in GaAs IMPATT diodes with ultrathin $i$-zones is triggered by Zener tunneling in the high field region, whereas the generation process itself starts within the depletion layers. This leads to a pronounced dark-space effect in the impact ionization rates. For applied voltages below $5.7 \mathrm{~V}$, this carrier generation cycle does not lead to breakdown. However, it takes almost 30 ps after switch-on before the current is fully stationary when the applied bias is close to this breakdown threshold value.

Since the impact ionization rate and the drift velocity of electrons is higher than that of holes, the electrons get more efficiently cooled on the $n$ side of the intrinsic zone than the holes do on the opposite side. This leads to a slightly higher average energy of the holes, as well as to a higher density of holes on the $p$-side than electrons on the $n$-side. In the avalanche regime, the higher hole density effectively screens the electric field in the intrinsic zone. This leads to an interesting effect for short $i$-zones of less than $50 \mathrm{~nm}$. Once the reverse bias exceeds the threshold value for avalanche multiplication, the holes are able to flood the whole $i$-zone. This screens the electric field and effectively impedes the avalanche breakdown.

\subsection{Quantitative Results and Comparison with Data}

To substantiate this qualitative picture, Figures 4(a)-4(d) characterize the carrier distribution for a reverse bias of $5.5 \mathrm{~V}$ in the $20 \mathrm{~nm} p$-i- $n$ diode (i.e., just below breakdown) quantitatively. The spatial field profile is drawn as grey inset in these figures in order to show the spatial extent of the depletion zone. The electric field is constant within the intrinsic zone and amounts to $1.1 \mathrm{MV} / \mathrm{cm}$.

The energy of electrons (Fig. 4(a)) reaches a maximum at the center of the high field zone. There, the electrons loose energy predominantly by impact ionization. Electrons are able to fly a longer distance than holes before they loose energy and pick up a given amount of energy on a shorter trajectory. Therefore, the average electron energy

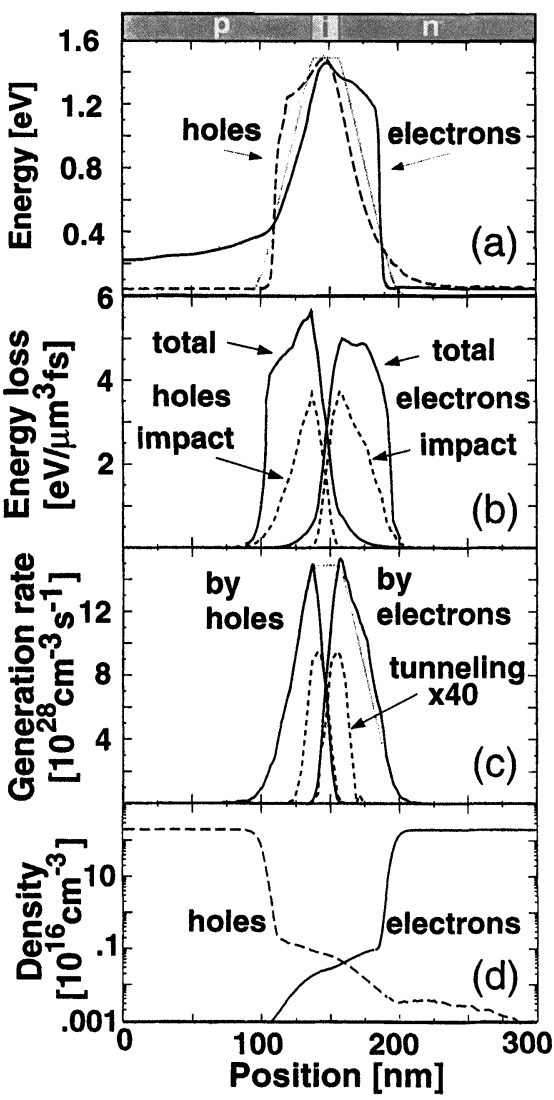

FIGURE 4 The figure refers to a $20 \mathrm{~nm}$ PIN diode with a doping level of $2 \times 10^{18} \mathrm{~cm}^{-3}$ in the $n$ - and p-regions, respectively, and an applied reverse bias of $5.5 \mathrm{~V}$. The shape of the electric field is shown in grey to indicate the width of the depletion zone. Its magnitude is $1.1 \mathrm{MV} / \mathrm{cm}$. (a) Calculated average energy of electrons and holes as a function of position. (b) Calculated energy loss rate of electrons and holes. Shown are the total energy losses and the contributions from impact ionization. (c) Calculated impact ionization generation rate of electron hole pairs induced by electrons and holes, respectively (full lines). Zener generation rate of electrons and holes, respectively (dashed lines). (d) Calculated density of electrons and holes.

decreases more slowly and extends farther into the depletion region on the $n$-side than the hole energy does on the $p$-side.

The same effect can be deduced from the spatially resolved energy loss (Fig. 4(b)). The energy loss by impact ionization is higher for electrons than for holes and remains large throughout the depletion region on the $n$-side. Since electrons gain energy more rapidly than 
holes, they are more likely to impact ionize repeatedly. The slower holes, on the other hand, remain hotter which leads to their higher total energy loss. Particularly near the end of the $p$-sided depletion region, their energy loss is dominated by phonon and plasmon scattering. These results are consistent with the higher integral of the electron induced electron-hole pair generation rate that is shown in Figure 4(c). This figure also includes the number of generated electrons and holes by Zener tunneling. The latter generation process gives a negligible contribution to the current within $0.2 \mathrm{~V}$ of breakdown.

The higher electron-induced impact ionization rate generates more holes near the $i-n$ junction than the opposite process generates electrons near the $p-i$ region. Therefore, the density of minority carriers is higher on the $n$-side than on the $p$-side (Fig. 4(d)). This effect is enhanced by the higher mobility of electrons that causes them to diffuse out of the $p$-zone more rapidly. The asymmetry of carrier densities within the high field region that can be seen in Figure 4(d) is another consequence of the higher impact scattering rate for electrons. The holes that are generated by impact ionization on the $n$-side get accelerated through the high field region towards the $p$-zone, leading to the excess density of holes on the $p$-side.

Figure 5 compares the present calculations with the measured current-voltage characteristics for a $20 \mathrm{~nm}$ and $50 \mathrm{~nm}$ pin diode, respectively. The agreement is seen to be very good. In Figure 6, we predict the I-V characteristics of $30 \mathrm{~nm} \mathrm{AlGaAs} p$ $i-n$ structures. Since the energy gap is higher than in GaAs, the impact ionization rate is lower which causes the breakdown voltage to increase and the current density to decrease with increasing $\mathrm{Al}$ concentration. The lower breakdown voltage compared to the $20 \mathrm{~nm}$ diode discussed above is caused by the higher doping level of the $n$ and $p$ regions.

The work has been partially supported by SIEMENS and by the Deutsche Forschungsgemeinschaft (SFB 384).

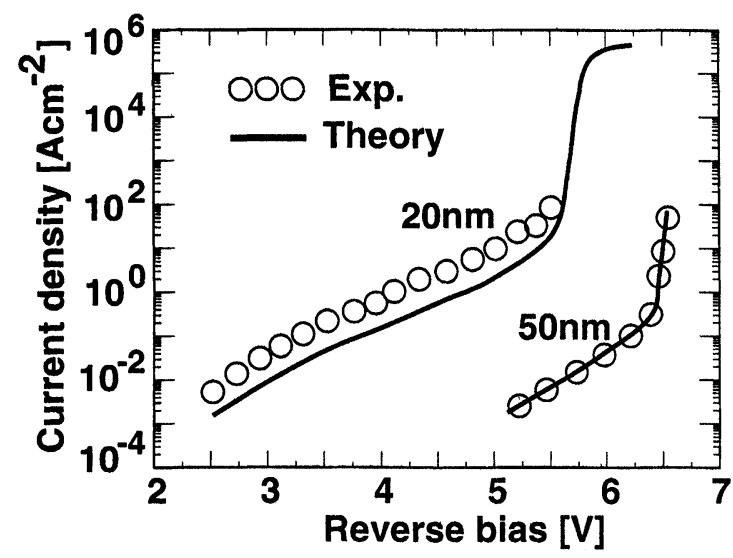

FIGURE 5 Calculated (full curves) and experimental current densities (stars), in $\mathrm{A} / \mathrm{cm}^{2}$, versus applied reverse bias in $\mathrm{V}$ for a $20 \mathrm{~nm}$ and $50 \mathrm{~nm}$ GaAs $p-i-n$ diode. The doping concentration in the $n$ and $p$-layers amounts to $2 \times 10^{18} \mathrm{~cm}^{-3}$. The experimental data are from [6].

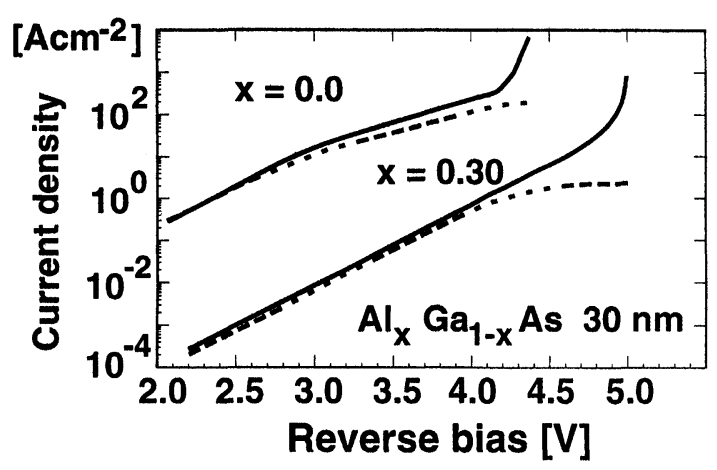

FIGURE 6 Predicted current densities versus applied reverse bias for a $30 \mathrm{~nm} \mathrm{GaAs}$ and $\mathrm{Ga}_{0.7} \mathrm{Al}_{0.3} \mathrm{As} p$ - $i-n$ diode. The doping concentration in the $n$ and $p$-layers is $5 \times 10^{18} \mathrm{~cm}^{-3}$. The dotted lines show the Zener tunneling contribution to the current density.

\section{References}

[1] Kometer, K., Zandler, G. and Vogl, P. (1992). "Latticegas cellular-automaton method for semiclassical transport in semiconductors", Phys. Rev. B., 46, 1382-1394.

[2] Zandler, G., Di Carlo, A., Kometer, K., Lugli, P., Vogl, P. and Gornik, E. (1993). "A comparison of Monte Carlo and cellular automaton approaches for semiconductor device simulations", IEEE Electron Dev. Letters, 14, 77-79.

[3] Rein, A., Zandler, G., Saraniti, M., Lugli, P. and Vogl, P. (1994). "Cellular Automaton Simulations of Planar Doped Barrier Field Effect Transistor", Proceedings of the 3rd International Workshop on Computational Electronics (S. M. Goodnick, ed.), 7-10, Oregon State University Press, Corvallis. 
[4] Zandler, G., Saraniti, M., Rein, A. and Vogl, P. (1996). "Cellular Automata for Device Simulation E Concepts and Applications", Proceedings of 1996 Int. Conf. on Simulation of Semiconductor Processes and Devices, 3942, (Business Center for Academic Societies Japan), Tokyo, ISBN 0-7803-2745-4.

[5] Vogl, P., Zandler, G., Rein, A. and Saraniti, M. (1997). "Cellular Automaton approach for semiconductor transport", in Theory of Transport Properties of Semiconductor Nanostructures (E. Schoell, ed.), Chapman and Hall, London, in print.

[6] Gaul, L., Huber, S., Freyer, J. and Claassen, M. (1991). "Determination of tunnel-generation rate from GaAs PIN-structures", Solid State Electron., 34, 723-726.

[7] Poebl, M., Bogner, W. and Gaul, L. (1994). "CW GaAs MITATT Source on Copper Heatsink up to $160 \mathrm{GHz}$ ", Electronic Letters, 30, 1316-1317.

[8] Tschernitz, M. and Freyer, J. (1995). "140 GHz GaAs Double-Read IMPATT Diodes", Electronic Letters, 31, $582-583$.

[9] Liebig, D., Lugli, P., Vogl, P., Claasen, M. and Harth, W. (1992). "Tunneling and ionization phenomena in GaAs PIN diodes", Microelectronic Engineering, 19, 127-130.

[10] Canali, C., Pavan, P., Di Carlo, P., Lugli, P., Malik, R., Manfredi, M., Neviani, A., Vendrame, E., Zanoni, E. and Zandler, G. (1996). "Experimental and Monte Carlo analysis of impact-ionization in AlGaAs/GaAs HBT's", IEEE Trans. on Elec. Dev., 43, 1769-1777.

[11] Kane, E. O. (1967). "Electron scattering by pair production in silicon", Phys. Rev., 159, 624-631.

[12] Krieger, J. B. (1996). "Theory of tunneling in semiconductors with dgenerate band structure", Ann. Phys., 36, 1-60.

[13] Ando, H. and Kanbe, H. (1981). "Ionisations coefficient measurements in GaAs by using multiplication noise characteristics", Solid State Electron., 24, 629-634.

[14] Bulman, G. E., Robbins, V. M., Brennan, K. F., Hess, K. and Stillman, G. E. (1983). "Experimental determination of Impact ionization coefficients in (100) GaAs", IEEE Electron Dev. Lett., 4, 181-185.

[15] Robbins, V. M., Smith, S. C. and Stillman, G. E. (1988). "Impact ionization in $\mathrm{Al}_{x} \mathrm{Ga}_{1-x}$ As for $x=0.1-0.4$ ", Appl. Phys. Lett., 52, 296-298.

\section{Authors' Biographies}

Günther Zandler is research associate at the Walter Schottky Institute of the Technical University of Munich and deals with high field transport and ultrafast phenomena.

Ralph Oberhuber is doing his $\mathrm{Ph} . \mathrm{D}$. thesis at the Technical University in Munich on new device concepts.

Dennis Liebig is doing his Ph.D. thesis at the Technical University in Hamburg-Harburg on device modeling.

Peter Vogl is professor of physics at the Technical University of Munich. He is interested in the theory of electronic structure and carrier transport in solids.

Marco Saraniti is post-doctoral fellow at ASU in Tempe. He focuses on numerical aspects of the simulation of electron devices.

Paolo Lugli is professor of optoelectronics at the 2nd University of Rome and focuses on semiconductor device modeling and ultrafast phenomena. 

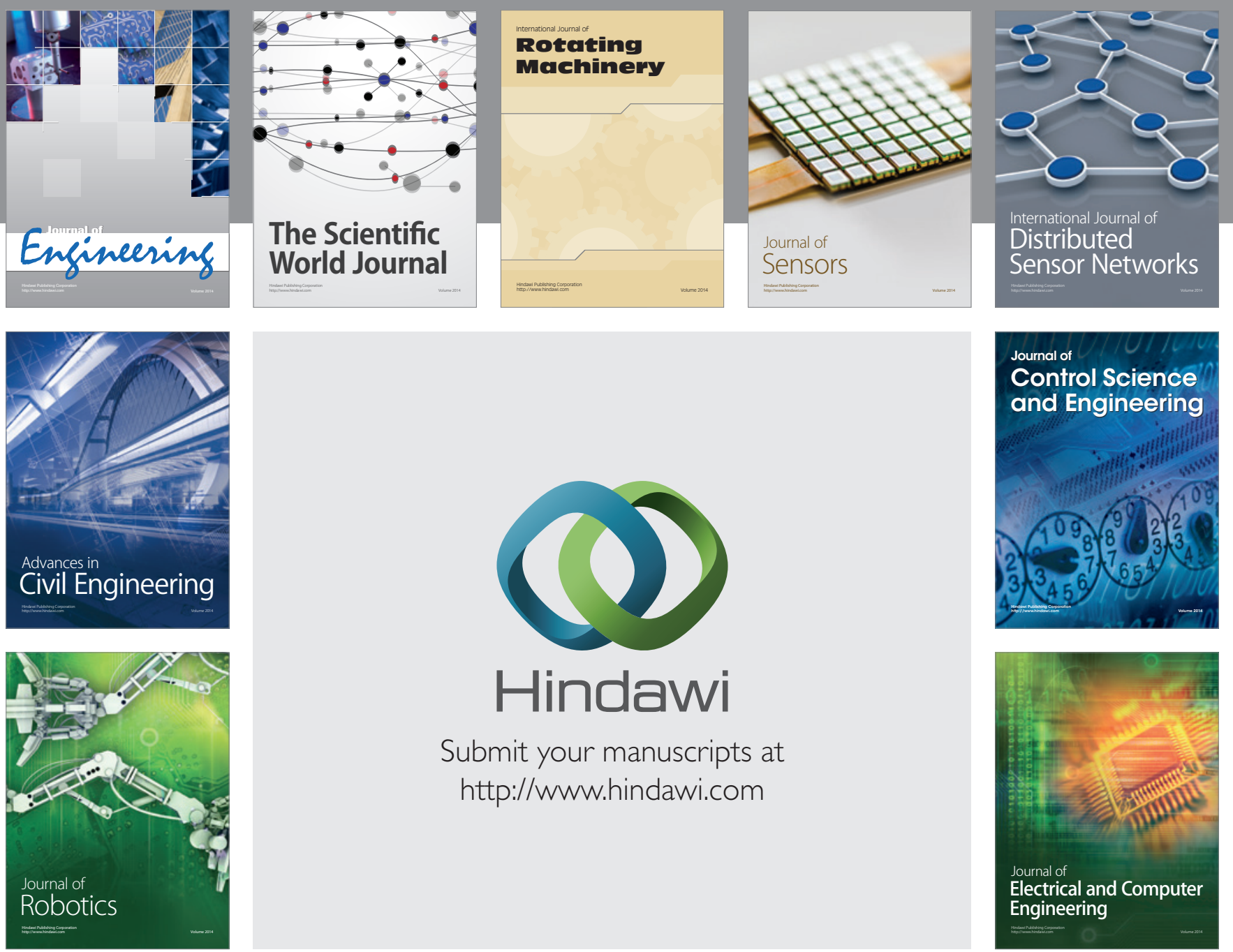

Submit your manuscripts at

http://www.hindawi.com
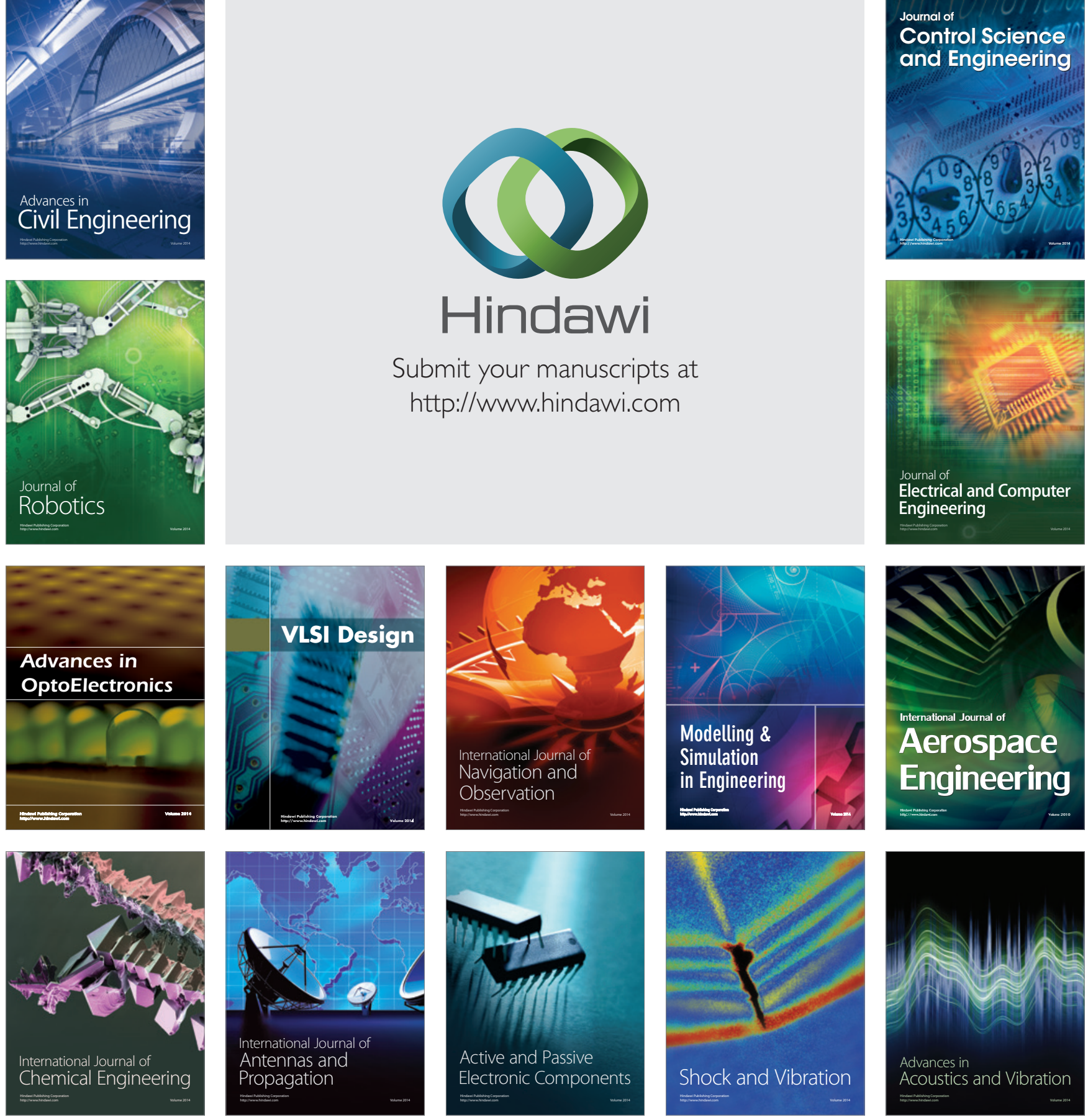